[Radiocarbon, Vol. 15, No. 1, 1973, P. 299-239]

\title{
UNIVERSITY OF WISCONSIN RADIOCARBON DATES $X$
}

\author{
MARGARET M. BENDER, REID A. BRYSON, and \\ DAVID $A$. BAERREIS
}

Department of Meteorology

University of Wisconsin, Madison

Radiocarbon dates obtained since December, 1970, are summarized here. Procedures and equipment have been described previously ( $R$., I966, v. 8, p. 522). Wood, charcoal, and peat samples are pretreated with dilute $\mathrm{NaOH}$ and dilute $\mathrm{H}_{3} \mathrm{PO}_{4}$ belore conversion to the counting gas, methane; marls and lake cores are treated with acid only. Very calcareous materials are treated with $\mathrm{HCl}$ instead of $\mathrm{H}_{3} \mathrm{PO}_{4}$.

The dates reported have been calculated using 5568 as the halflife of $\mathrm{C}^{14}$, with 1950 as the reference year. The standard deviation quoted includes only $l \sigma$ of the counting statistics of background, sample, and standird counts. Methane prepared from NBS oxalic acid is used as the standard; the $C_{1}^{13} / C_{1}$ ratios of the $C_{0}$. prepared from this oxalic acid are measured and the activity of the standard methane is corrected for any deviation of the $\delta \mathrm{C}^{13}$ value of the $\mathrm{CO}_{2}$ sample from the $-19 \%$ value (compared to the PDB standard) reported by Craig (I961). The dated samples for which $\delta \mathrm{C}^{13}$ values are listed have been corrected to $-25 \%$ (PDB stanclard), the "normal" value of terrestrial material.

\section{ACKNOWLEDGMENTS}

This research is supported by the National Science Foundation, Atmospheric Sciences Division, Grant GA-10651X. We thank the Chemistry Department for the use of the Nuclide RMS-660 mass spectrometer and Roger Arhart for technical assistance with the mass spectrometer.

\section{ARCHAGOLOGL: SAMPLES}

\section{A. Illinois}

\section{WIS-474. Divers site (MO-28)}

$1010 \pm 55$

Wood, specimen 319, from Feature 36, SW wall of house, 2nd burned house from Divers site, Monroe Co., Illinois $\left(38^{\circ} 27^{\prime} 42^{\prime \prime} \mathrm{N}^{r}\right.$ Lat, 90 15' 25" W Long). Previous date, A.D. I I05, WIS-334, was reported earlier (R., 1970, v. 12, p. 340).

\section{Cahokia series}

Charcoal from E of Monks Mound, Cahokia Mounds State Park, Madison Co., Illinois (38 $48^{\prime} \mathrm{N}$ Lat, $90^{\circ} 04^{\prime} \mathrm{W}$ Long), and from Mound 72. Callokia Mounds State Park, St. Clair Co., Illinois (38 $39^{\prime} \mathrm{N}$ Lat, $90^{\circ} 04^{\prime} \mathrm{W}$ Long). Coll. 1966 to 1970 by Melvin Fowler and J. B. Anderson; subm. by Melvin Fowler, Univ. Wisconsin-Milwaukee.

WIS-492. Cahokia

$900 \pm 55$

UWM Sample 70-23 from Mound 72, Feature 227. Feature is large, 
irregular, shallow mound pit assoc. with earliest construction activity at Mound 72 .

\section{WIS-494. Cahokia}

$900 \pm 55$

UWM Sample 66-559 from structural timber, N199-200, E454-456, ca. $154 \mathrm{~m} \mathrm{E}$ of Monks Mound. Structure preceded all phases of stockade construction E of Monks Mound. Sample directly assoc. with classic Ramey Incised pot.

\section{WIS-495. Cahokia}

$850 \pm 50$

UWM Sample 67-1703, timber from Structure 4, N315.80-315.90, E466.90-467.05, ca. $166 \mathrm{~m} \mathrm{E}$ of Monks Mound. Sample from floor of House 4, destroyed witl contents in place. Ceramic inventory includes 2 variants of Ramey Incised, weeping eye design, and 1 cord marked vessel. Structure preceded all phases of stockade construction E of Monks Mound.

\section{WIS-493. Cahokia}

UMW Sample 67-387, support beam for House 4, N315.38-315.48, E467.45-467.62, $166 \mathrm{~m} \mathrm{E} \mathrm{of} \mathrm{Monks} \mathrm{Mound.}$

\section{Cahokia, Monk's Mound}

Charcoal from 4th terrace of Monk's Mound, Cahokia Mounds State Park, Madison Co., Illinois (38 $40^{\prime} \mathrm{N}$ Lat, 90 $04^{\prime} \mathrm{W}$ Long). Coll. 1970 by Univ. Washington, St. Louis, party dir. by Nelson Reed.

\section{WIS-525. Monk's Mound}

Sample from Fcature 1B.

\section{WIS-527. Monk's Mound}

Sample from Feature $1 \mathrm{H}$.

\section{WIS-528. Monk's Mound}

Sample from Post 6, Feature 26.

\section{B. Iowa}

$870 \pm 55$ A.D. 1080 $\delta C^{13}=-28.4 \%$ $890 \pm 60$
A.D. 1060
$\delta C^{1 *}=-26.5 \%$

$$
\begin{aligned}
& 970 \pm 65 \\
& \text { A.D. } 980 \\
& \delta C^{13}=-28.3 \%
\end{aligned}
$$

\section{Brewster site series}

Excavations conducted during the summer of 1970, sponsored by Univ. Wisconsin-Madison and Sanford Mus., Cherokee, Iowa, at the Brewster site (13GK15) (420 $49^{\prime} \mathrm{N}$ Lat, $95^{\circ} 36^{\prime} \mathrm{W}$ Long) resulted in twodirectional sectioning of the midden. Dates from charcoal specimens at various depths imply relatively thick midden was constructed in brief 
time period and that excavation of house pits and features clisturbed the continuity of deposition. Subm. by 1). A. Bacreis.

WIS-496. Brewster site (13CK15)

A.D. 1075

$875 \pm 60$

Charcoal from Level 1 to 2,15 to $25 \mathrm{~cm}$ depth, Sq. $530 \mathrm{~W} 60$.

\section{WIS-500. Brewster site (13CK15)}

$1020 \pm 55$ A.D. 930

$\delta C^{1.3}=-26.5 \%$

Charcoal from Level 1 and 2, Sq. S30W70, Sample 191, and Sample 182 from Sq. S50W40, 20 to $30 \mathrm{~cm}$ deep.

WIS-468. Brewster site (I3CK15)

$925 \pm 75$

Sample from I evel 1, 20 to $30 \mathrm{~cm}$ depth, Sq. S25W85.

WIS-480. Brewster site (13CK1.5)

Charcoal from Level 1, Sq. S30W85.

Che

WIS-453. Brewster site (13CK15)
$990 \pm 55$
A.D. 960

$\mathbf{7 5 0} \pm \mathbf{5 0}$

Sample from Level 3 to $4,30.5$ to $41 \mathrm{~cm}$ depth, Sq. S25W85.

W1S-469. Brewster site (13CK15)

$810 \pm 70$

Charcoal from Level $1 \mathrm{l}$ to 12,71 to $81 \mathrm{~cm}$ below surface, Sq. S25W90.

W IS-156. Brewster site (13CK15)

$925 \pm 55$

Sample from Level 15 to 16,81 to $91 \mathrm{~cm}$ deep, from Sq. S25W90.

WIS-463. Brewster site (13CK15)

Sample from Level 15 to 16 , Sq. S25W85.

WIS-473. Brewster site (13CK15)

A.D. 1025

$950 \pm 55$

A.D. 1000

Sample from Level 16 to 17,91 to $102 \mathrm{~cm}$ deep, Sq. S30W90, and Level 15,91 to $97 \mathrm{~cm}$ deep, Sq. S25W90.

WIS-482. Brewster site (13CK15)

A.D. 1000

$950 \pm 55$

Sample from Level 19 to 20,112 to $192 \mathrm{~cm}$ deep, Sq. S25W85.

WIS-511. Brewster site (13CK15)

$1015 \pm 55$

Chatrcual from Level 21 to 29,122 to $132 \mathrm{~cm}$ deep, Sq. S25W85.

WIS-461. Brewster site (13CK15)

$1010 \pm 55$

Sample from Level 25 to 26 , 14 2 to $152 \mathrm{~cm}$ depth, Sq. S30W85. 
WIS-464. Brewster site (13CK15)

$1000 \pm 55$

Sample from Level 25 to 26, Sq. S30W90.

A.D. 950

WIS-505. Brewster site (13CK15)

Sample from Level 25 to 26 , Sq. S25W90.

A.D. 1040

$910 \pm 60$

WIS-497. Brewster site (13CK15)

$1025 \pm 55$

A.D. 925

Sample from Level 29 to 30,160 to $170 \mathrm{~cm}$ deep, Sq. S35W90.

\section{Meehan-Schell site (13BNI10)}

Charcoal from Mechan-Schell site, Boone Co., Iowa (Saylorville Reservoir) (42 $2^{\prime} 0^{\prime \prime} \mathrm{N}$ Lat, $93^{\circ} 56^{\prime} 50^{\prime \prime} \mathrm{W}$ Long). Coll. 1970 by David Gradwohl, Iowa State Univ.; subm. by D. A. Baerreis. Site is Great Oasis component.

\section{WIS-501. Meehan-Schell site (13BN110)}

$$
\begin{aligned}
& \mathbf{8 7 0} \pm \mathbf{6 0} \\
& \text { A.D. } 1080 \\
& \delta C^{13}=-28.1 \%
\end{aligned}
$$

Catalogue no. 2714 and 2726 from Feature 35, storage pit.

WIS-502. Meehan-Schell site (13BN110)

Catalogue no. 2677 from Feature 32, storage pit.

WIS-498. Meehan-Schell site (13BN110)

Catalogue no. 2849 from Feature 37, storage pit.
$975 \pm 55$

A.D. 975

$\delta C^{13}=-27.2 \%$

A.D. 1000

$$
950 \pm 55
$$

Sparks site (13BN121)

Charcoal from Sparks site, Boone Co., Iowa $\left(42^{\circ} 2^{\prime} 0^{\prime \prime} \mathrm{N}\right.$ Lat, $93^{\circ}$ $56^{\prime} 30^{\prime \prime}$ W Long). Coll. 1970 by David Gradwohl; subm. by D. A. Baerreis.

\section{WIS-517. Sparks site (13BN121)}

$1600 \pm 55$

Charcoal from Feature 19 and Feature 13, storage pits or basins.

\section{Broken Kettle West site (13PM25)}

Charcoal excavated 1969, dir. by D. R. Henning, Univ. Nebraska,

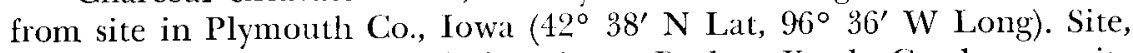
of Great Oasis cultural affiliation, is on Broken Kettle Creek, opposite Broken Kettle midden (13PM1) of Mill Creek cultural affiliation. See dates of $13 \mathrm{PM} 1$, below, which suggest considerable overlap in the 2 occupations.

WIS-433. Broken Kettle West site (13PM25)

$$
1070 \pm 55
$$

Sample 260 from House 3, Pit 16, Area 1. 
WIS-439. Broken Kettle West site (13PM25) $1090 \pm 55$

Sample 89 from entrance of House 2, Area 1.

WIS-440. Broken Kettle West site (13PM25) A.D. 860

Sample 396 from House 3, Pit 18, Area 1.

WIS-451. Broken Kettle West site (13PM25)

$$
1100 \pm 50
$$

A.D. 850

Sample 168 from House 2, Pit 5, Area 1, Feature 5.

WIS-455. Broken Kettle West site (13PM25) A.D. 1010

$$
840 \pm 55
$$

A.D. 1110

Sample 410 from House 3, Pit 19.

WIS.452. Broken Kettle West site (13PM25) A.D. 1070

$$
880 \pm 55
$$

Sample 425 from House 3, Pit 25.

WIS-488. Broken Kettle West site (13PM25)

A.D. 1060

$890 \pm 55$

Sample 425, fresh preparation.

WIS-481. Broken Kettle West site (13PM25) $\delta C^{13}=-26.4_{\% 0}^{\circ}$

Sample 132 from House 2 entrance.

WIS-499. Broken Kettle West site (13PM25)

$$
980 \pm 55
$$

A.D. 970

$\delta C^{13}=-26.5 \%$

Sample 409 from House 3, Area 1, Pit 25.

\section{Broken Kettle site, Iowa (13PM1)}

Charcoal from Broken Kettle site, Plymouth Co., lowa $\left(42^{\circ} 38^{\prime} \mathrm{N}\right.$ Lat, $96^{\circ} 36^{\prime} \mathrm{W}$ Long). Coll. 1969 by D. R. Henning; subm. by D. A. Baerreis. Earlier dates from this site were previously reported (R., 1968, v. 10, p. 474$)$.

WIS-478. Broken Kettle site (13PMI)

$990 \pm 45$

Sample from Sq. 10E10S, Level 8, 107 to $122 \mathrm{~cm}$ deep, Feature B.

WIS-484. Broken Kettle site (13PM1)

$$
900 \pm 45
$$

Specimen 8 from Sq. 10E10S, Level 10, 137 to $152 \mathrm{~cm}$ deep.

WIS-506. Broken Kettle site (13PMI)

A.D. 1080

$$
870 \pm 50
$$

Specimen 8a from Feature E, Level 10,137 to $152 \mathrm{~cm}$ deep.

WIS-485. Broken Kettle site (13PM1)

$925 \pm 55$

Sample from Feature H, Sq. 15E5S.

A.D. 1025 
WIS-476. Broken Kettle site (13PM1)

$\mathbf{9 5 0} \pm \mathbf{5 0}$
A.D. $\mathbf{1 0 0 0}$
$\delta C^{13}=-27.0 \%$

Charcoal from Sq. 10E10S, Level 11, 152 to $168 \mathrm{~cm}$ below surface, Specimen 40.

WIS-479. Broken Kettle site (13PM1)

$960 \pm 55$

Specimen 113 from Sq. 10E10S, Level 13, 183 to $198 \mathrm{~cm}$ deep.

WIS-531. Broken Kettle site (13PM1)

A.D. 1165

$785 \pm 60$

Sample 73 from I.evel 16, Sq. 15E5S, Fcature I, 229 to $244 \mathrm{~cm}$ deep.

WIS-477. Broken Kettle site (13PMI)

A.D. 1010 deep.

Specimen 97 from Sq. 10L5S, Feature K, Level 2, 231 to $279 \mathrm{~cm}$

WIS-530. Broken Kettle site (13PMI)

A.D. 1040

$910 \pm 60$

Sample 77 from Level 17, Feature H, Sq. 15E5S, 244 to $259 \mathrm{~cm}$ deep.

WIS-503. Broken Kettle site (13PM1)

$955 \pm 60$

Sample 87 from Feature K, Sq. 10E5S, 279 to $287 \mathrm{~cm}$ decp.

\section{McKinstry Mounds (21KC2)}

\section{Minnesota}

Samples from McKinstry Mound 1 at mouth of Little Fork R.,

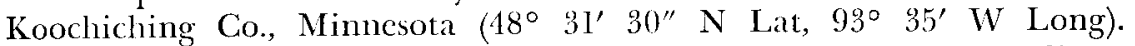
Coll. 1970 and subm. by J. B. Stoltman, Univ. Wisconsin-Madison. Burial mound is Middle Woodland, assigned to Laurel culture of $\mathrm{N}$ Minnesota.

WIS-471. MeKinstry Mounds (21KC2)

$1700 \pm 55$

Unburned wood from $\log$ floor at base of mound, Level 16 in Sq. T12.

WIS-486. MeKinstry Mounds (21KC2)

$1980 \pm 45$

30 B.C.

Charcoal from "Basal black" level of mound, the margins of 1st stage of mound construction of Mound 1. Sample from Feature 23, Sq. S11, Level 11b. WIS-471 lay at base of this layer.

WIS-487. McKinstry Mounds (21KC2)

$$
1940 \pm 60
$$

Charcoal from 2nd stige of mound construction, Mound A. Sample from Feature 21, Sq. T12, Level 16. 
WIS-489. MeKinstry Mounds (21KC2)

$1830 \pm 55$

Charcoal from Mound B, 3rd constructional stage in mound. Combined sample from Faature 24, Sq. S12, Level 10 and Level 11, and Feature 21, Sq. T12, Level 15 .

WIS-490. McKinstry Mounds (21KC2)

$1390 \pm 55$

Charcoal from Mound $\mathrm{C}$, 1th stage of mound construction, from Feature 17, Sq. S10, Level 4.

\section{Great Oasis culture, Minnesota}

Specimens coll. 1971 by Univ. Minnesota field school, supervised by D. R. Henning. Study involved a reinvestigation of Great Oasis components previously reported by I. A. Wilford, Univ. Mimnesota (Wilford, $1945 ; 1955)$. Subm. by 1). A. Baereis.

\section{WIS-522. Thompson site (21MU17)}

$$
\begin{aligned}
& \mathbf{1 0 5 0} \pm \mathbf{6 0} \\
& \text { A.D. } 900 \\
& \delta C^{13}=-26.5 \% \mathrm{co}
\end{aligned}
$$

Charcoal coll. 1971 from Murray Co., Minnesota (44 $5^{\prime} \mathrm{N}$ Lat, $95^{\circ}$ $55^{\prime} 30^{\prime \prime}$ W Long). Sample 53 from Feature 2, Sq. 3, 61 cm deep.

WIS-532. Low Village site (21MU2)

$975 \pm 65$

Samples 17 and 18 from Feature C, Sq. 1,38 to $51 \mathrm{~cm}$ deep, in

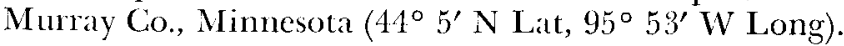

\section{Over focus series}

\section{South Dakota}

Dating of samples from Mitchell site (39DV2), the type site of the Over focus in South Dakotat, during July-August, 1971, are combined with an examination of samples from earlier work in order to clarify the temporal position of this cultural unit. The long-rectangular house assemblage is considered one of earliest scdentary village complexes of this region.

\section{Mitchell site (39DV2), South Dakota}

Excavations were made in 1971 by a Univ. Wisconsin field party, supervised by R. A. Alex at Mitchell site $\left(43^{\circ} 43^{\prime} \mathrm{N}\right.$ Iat, $98^{\circ} 02^{\prime} \mathrm{W}$ Long), Davison Co., South Dakota. 'The site contains only single cultural component and was previously tested by E. E. Meleen in 1938 (Meleen, 1938). Samples subm. by D. A. Baerreis.

WIS-509. Mitchell site (39DV2)

Charcoal from Feature 6, Sq. J, 30 to $46 \mathrm{~cm}$ deep. 
WIS-510. Mitchell site (39DV2)

$960 \pm 55$

A.D. 990

$\delta C^{13}=-25.5 \%$

Charcoal from Feature 5, Level 9, 122 to $142 \mathrm{~cm}$ deep.

WIS-512. Mitchell site (39DV2)

$965 \pm 50$

A.D. 985

Charcoal from House 3, Sq. I.

$\delta C^{13}=-25.7 \%$

WIS-514. Mitchell site (39DV2)

$890 \pm 55$

A.D. 1060

$\delta C^{13}=-25.6 \%$

Charcoal from House 4, Sq. U, E half, 46 to $55 \mathrm{~cm}$ below surface.

WIS-518. Mitchell site (39DV2)

$\mathbf{9 1 0} \pm \mathbf{5 5}$
A.D. 1040
$\delta C^{13}=-25.4 \%$

Charcoal from House 4, Sq. W, E half, wall daub layer, 55 to 70 cm below surface.

\section{WIS-521. Mitchell site (39DV2)}

$950 \pm 55$

Charcoal from Sq. U, SE corner, wall post, $46 \mathrm{~cm}$ deep.

\section{Swanson site (39BR16), South Dakota}

The Swanson site (43 $54^{\prime} \mathrm{N}$ Lat, $99^{\circ} 20^{\prime} \mathrm{W}$ Long), Brule Co., South Dakota is an Over focus component excavated 1950 by W. R. Hurt, Jr. (1951). A single date, A.D. $850 \pm 250$, M-839, was obtained from wood from Post G, House 2 (Grane and Griffin, 1960). A series of additional posts from the site, preserved at the $W$. H. Over Mus. in Vermillion, South Dakota, were obtained for dating through the courtesy of J. S. Sigstad, Univ. South Dakota and W. R. Wood, Univ. Missouri; subm. by D. A. Bacreis.

\section{WIS-524. Swanson site (39BR16)}

Wood from outer rings of Post D, House 2.

WIS-526. Swanson site (39BR16)

Wood from outer rings of Post C, House 2.

WIS-523. Swanson site (39BR16)

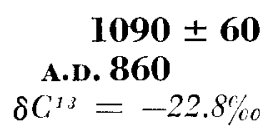

A.D. 1025

$$
925 \pm 55
$$

$\delta C^{13}=-23.2 \%$

$\mathbf{1 4 5 0} \pm \mathbf{6 0}$
A.D. 500
$\delta C^{1 s}=-21.9 \%$ o

Wood from outer rings of Post 2, House 1. Date is inconsistent and 
suggests sample was contaminated, perhaps by preservative, in museum storage. See WIS-529.

\section{WIS-529. Swanson site (39BR16)}

Sample from inner rings of Post 2, House 1.

$$
\begin{gathered}
1190 \pm 70 \\
\text { A.v. } 760 \\
\delta C^{13}=-22.8 \%
\end{gathered}
$$

$$
730 \pm 55
$$

WIS-513. Breeden site (39ST16)

Charred grass from the Breeden site $\left(44^{\circ} 25^{\prime} \mathrm{N}\right.$ Lat, $100^{\circ} 23^{\prime} 33^{\prime \prime}$ W Long) Stanley Co., South Dakota. Coll. 1955 by R. P. Wheeler; subm. by D. A. Baerreis. Date, A.D. $710 \pm 150$, M-608, (Crane and Griflin, 1960) was previously reported from this site.

\section{GEOLOGIC: SAM PLES}

\section{Willard Cave}

\section{A. Iowa}

Bone from Willard Cave, $8.4 \mathrm{~km} \mathrm{E}$ of Edgewood, Delaware Co., Iowa (42 $38^{\prime} 15^{\prime \prime} \mathrm{N}$ Lat, $91^{\circ} 17^{\prime} 30^{\prime \prime} \mathrm{W}$ Long). Coll. 1970 by R. E. Eshelman, Univ. Iowa, Iowa City; subm. by D. A. Baerreis. Present area of sympatry of $2 \mathrm{id}$. taxa from this cave is $483 \mathrm{~km}$ to NW of Delaware Co., based on presence of Clethrionomys gapperi, the boreal red-backed vole and Onychomys lencogaster, the N grasshopper mouse.

\section{WIS-483. Willard Cave}

Bone of small animals from $168 \mathrm{~cm}$ below surface of $\mathrm{S}$ talus from Level 6,76 to $91 \mathrm{~cm}$ level.

\section{WIS-491. Willard Cave}

$$
\begin{array}{r}
\mathbf{1 2 5 5} \pm \mathbf{5 5} \\
\text { A.p. } 695 \\
\delta C^{13}=-21.1 \%
\end{array}
$$

Bones (Odocoileus virginianus) from small cavity $259 \mathrm{~cm}$ below surface of $S$ talus slope, but slumping of talus deposits may have covered younger material clerived from different cave entrance.

\section{B. Wisconsin}

\section{WIS-508. Green Bay Campus Wood}

$11,940 \pm 110$ 9990 B.C.

Small branch from trench excavated 1971, Univ. Wisconsin-Green Bay campus, Brown Co., Wisconsin (44 $32^{\prime} \mathrm{N}$ Lat, $87^{\circ} 55^{\prime} \mathrm{W}$ Long) by Frank Byrne and Harry Guilford, Univ. Wisconsin-Green Bay. Wood, Two Creeks deposit, was $5.8 \mathrm{~m}$ below surface, lying beneath 4.6 $\mathrm{m}$ till (Valders) and above $30.5 \mathrm{~cm}$ brown and reddish clay above sand. 
Southwest Keewatin series

$$
\text { C. Canada }
$$

WIS-466. Kasmere Lake

$$
1265 \pm 55
$$

Charcoal from buried soil $15 \mathrm{~cm}$ below surface near base of esker. Coll. 1970 from Kasmere Lake, Manitoba, Canada $\left(59^{\circ} 40^{\prime} \mathrm{N}\right.$ Lat, $101^{\circ}$ $14^{\prime} \mathrm{W}$ Long) and subm. by C. J. Sorenson, Univ. Wisconsin-Madison.

\section{WIS-470. Birch Bay}

$1085 \pm 45$

Charcoal from buried charcoal $30.5 \mathrm{~cm}$ below surface in matrix of fine sand near crest of esker near Birch Bay, N.W.T., Canada $\left(60^{\circ} 41^{\prime}\right.$ $\mathrm{N}$ Lat, $101^{\circ} 47^{\prime} \mathrm{W}$ Long). Coll. 1970 and subm. by C. J. Sorenson.

\section{WIS-472. Northwest Arm Ennadai}

$$
1520 \pm 55
$$

Charcoal from surface horizon of buried palcosol overlain by 5 to $10 \mathrm{~cm}$ more recent soil on S facing slope of esker on NW arm of Ennadai Lake, N.W.T., Canada ( $61^{\circ} 05^{\prime} \mathrm{N}$ Lat, $101^{\circ} 37^{\prime} \mathrm{W}$ Long). Coll. 1970 and subm. by C. J. Sorenson.

\section{Roundrock Lake, N.W.'T.}

Samples from 3 buried charcoal horizons overlying a stone line near top of an esker at Roundrock Lake, Mackenzie Dist., N.W.T. (64 ${ }^{\circ}$ $23^{\prime} \mathrm{N}$ Lat, $113^{\circ} 20^{\prime} \mathrm{W}$ Long). Site is in present forest/tundra ccotone and provides information on latitudinal migration of forest border during Holocene. Coll. 1971 and subm. by C. J. Sorenson and J. C. Knox, Univ. Wisconsin-Madison.

\section{WIS-519. Roundrock Lake, N.W.T.}

Wood from surface of buried paleosol, depth $102 \mathrm{~cm}$. Sample denotes climatic change toward more arctic conditions with attendant $S$ ward depression of trecline.

\section{WIS-515. Roundrock Lake, N.W.T.}

$1885 \pm 55$

Charcoal from buried layer of forest litter, depth $119 \mathrm{~cm}$. Date identifies an early period of climatic and vegetative change.

WIS-516. Roundrock Lake, N.W.T.

Charcoal, $152 \mathrm{~cm}$ deep, from buried forest remnant. Provides date for destruction by fire of a very early postglacial forest.

\section{WIS-520. Esker near Eileen Lake, N.W.T.}

Charcoal from upper horizon of buried podzol paleosol, depth 18 
cm, NNW of Eileen Iake, Mackenzie Dist., N.W.T. $\left(62^{\circ} 20^{\prime} \mathrm{N}\right.$ Lat, $107^{\circ} 47^{\prime} \mathrm{W}$ Long). Indicates period of climatic fluctuation in which forest gave way to mixed forest and tundra. Coll. 1971 and subm. by C. J. Sorenson and J. C. Knox.

\section{RIFERFNCFS}

Bender, M. M., Bryson, R. A., and Baerreis, D. A., 1966, University of Wisconsin radiocarbon dates II: Radiocarbon, v. 8, p. 522-533.

1968, University of Wisconsin radiocarbon dates V: Radiocarbon, v. 10, p. $473-478$

1970, University of Wisconsin radiocarbon dates VII: Radiocarbon, v. 12, p. $335-345$.

Craig, Harmon, 1961, Mass-spectrometer analyses of radiocarbon standards: Radiocarbon, v. 3 , p. 1-3.

Crane, H. R. and Griffin, J. B., 1960, University of Michigan racliocarbon dates V: Am. Jour. Sci. Radiocarbon Supp., v. 2, p. 31-48.

Hurt, W. R., Jr., 1951, Report of the investigation of the Swanson site, 39BR16, Brule County, South Dakota: Archaeological Studies, Circular No. 3. Pierre, South Dakota.

Meleen, E. E., 1938, A preliminary report of Mitchell Indian village site and burial mounds on Firesteel Creek, Davison County, South Dakota: Univ. of South Dakota Mus., archacol. studies cir., no. 2, pt. 1.

Wilford, L. A., 1945, Three village sites of the Mississippi pattern in Mimnesota: Am. Antiquitv, v. 11, no. 1, p. 39-40.

1955, A revised classification of the prehistoric cultures of Minnesota: Am. Antiquity, v. 2I, no. 2, p. 140-141. 